OPTOGENETICS

\section{Follow the PIF}

Dev. Cell 36, 117-126 (2016)

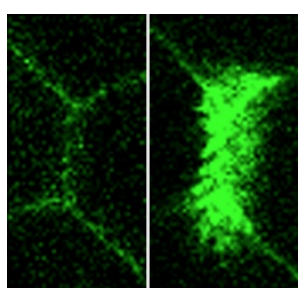

Experimental control of protein localization will help decipher protein function and manipulate cell behaviors. The phytochrome optogenetic system exploits the red light-mediated heterodimerization of the phytochrome B protein (PHYB) with its partner phytochrome interaction factor (PIF) in the presence of the chromophore PCB. However, this technique has not yet been applied to multicellular organisms because of the inability of PCB to penetrate deep tissues and the poor expression of PHYB. Buckley et al. were able to successfully optimize this system for zebrafish embryos by delivering a newly purified version of PCB into the embryos along with a truncated version of PHYB that optimizes its expression. When a PHYB-CAAX construct is used to drive membrane localization, exposure to 650-nm light rapidly recruits PIF6 to the membrane. Shifting the light to $750 \mathrm{~nm}$ reverses membrane binding of PIF6. Subcellular control of localization can be achieved by focusing the $650-\mathrm{nm}$ laser at a point of interest. Given their interest in the regulation of polarity during neurogenesis, the authors generated a Pard3-PIF6 fusion construct and were able to direct the apical polarity protein Pard 3 and recruit its binding partner Pard6 to specific membrane locations in the neural progenitors by the spot of the 650-nm laser. Overall, the use of this modified phytochrome system offers the potential to regulate cellular behaviors with greater control in a multicellular organism.

TARGET IDENTIFICATION

\section{Getting cholesterol out elife 4, e12177 (2016)}

Cholesterol transport within cells is a wellunderstood process wherein low-density lipoprotein (LDL) is internalized via receptormediated endocytosis and trafficked to lysosomes, where cholesterol is ultimately liberated. Cholesterol is then transported by a lysosome-lumenal protein called NPC2 to the lysosomal membrane-embedded cholesterol efflux transporter NPC1. A deficiency of $\mathrm{NPC1}$, as in the lysosomal storage disease Niemannn-Pick type C, leads to cholesterol accumulation within lysosomes. U18666A is a cationic amphiphile that inhibits the exit of LDL-derived cholesterol from lysosomes, mimicking NPC1 deficiency, though its target has not been formalized. To find the molecular target of U18666A, Lu et al. generated a photo-crosslinkable form of the compound, U-X, that also contained an alkyne group useful for a post-crosslinking attachment of functional elements such as fluorophores through click chemistry. Incubation of a $\mathrm{CHO}$ cell line with U-X identified a protein that was characterized as NPC1. The threshold for U18666A inhibition increased >100-fold with NPC1 overexpression, whereas NPC1 depletion resulted in a loss of $\mathrm{U}-\mathrm{X}$ crosslinking.

\section{RNA MODIFICATION}

\section{Translating for growth}

Testing several NPC1 point mutants that cause cholesterol transport deficiencies for their ability to crosslink with U-X led to the conclusion that U18666A binds to the sterolsensing domain (SSD) of NPC1, not the cholesterol-binding N-terminal domain. These results suggest that the SSD contains a second cholesterol-binding site that may act in a relay to move cholesterol from lysosomes. $M B$

\section{CATALYTIC DNA}

\section{Ligating with lambda \\ Nature 529, 231-234 (2016)}

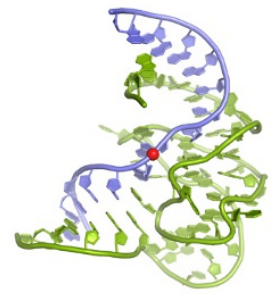

Enzymes comprised solely of DNA have not been observed in nature, but laboratory evolution experiments have identified proficient DNA catalysts. The lack of structural data on deoxyribozymes has limited our understanding of DNA-driven catalysis, but a new study reporting the X-ray crystal structure of the 9DB1 ligase deoxyribozyme, which catalyzes phosphodiester bond formation between the terminal 3'-hydroxyl and the $5^{\prime}$-triphosphate of RNA substrates, changes this landscape. In their 2.8- $\AA$-resolution structure of a 9DB1 DNA construct with a product RNA strand, Ponce-Salvatierra et al. revealed a $\lambda$-shaped folded complex, which is built from partially stacked short helical domains that are held in place by long-range base-pairing. Nucleotides of the A-G RNA ligation site are organized in a duplex-like conformation at the $\lambda$ three-way junction by specific base-pairing interactions with the protruding J2/3 region and additional stacking and tertiary interactions, which also enforce the enzyme's 3 ', 5 '-ligation regiochemistry. Unlike with ligase ribozymes, no catalytic metal ions were observed in the active site of 9DB1, but a phosphate oxygen of $\mathrm{dA} 13$ is positioned nearby and critical for ligation catalysis. Further functional group mutagenesis of key base and sugar residues validated the 9DB1 structural model, defined how the greater conformational flexibility of DNA nucleotides facilitates catalytic structure formation and informed rational design strategies for engineering 9DB1 for broader substrate specificity. 